\title{
Partial Discharges Measurements for Condition Monitoring and Diagnosis of Power Transformers: A Review
}

Johnatan M. Rodríguez-Serna Ricardo Albarracín-Sánchez Fernando Garnacho Fernando Álvarez Javier Ortego

\begin{abstract}
Power transformers are key electrical assets in power networks. Their lifetimes are estimated to be around $\mathbf{4 0}$ years if they are properly maintained. However, thermal, electrical and mechanical stresses can cause the apparition of partial discharge (PD) activity in the insulation system that can lead to its degradation. Thus, the presence of PD activity can be considered as indicator of failure. Conventional PD acquisitions can be done in accordance with IEC-60270 and IEC-60076-3 standards. In the last decades, unconventional methods, depicted in the IEC-TS-62478 standard, based on acoustic and electromagnetic measurements are being combined with the conventional ones for monitoring power transformers identifying and localizing PD sources. In this paper, the necessity of PD monitoring in power transformers is highlighted and a review of different methods for measuring PD is featured. Besides, some technological trends are depicted. Finally, PD modelling is presented as a promising compliment for PD diagnosis.
\end{abstract}

Index Terms-- Condition monitoring, electrical insulation, lifetime, partial discharge, power transformer, PD modelling.

\section{INTRODUCTION}

$\mathrm{L}$ IFETIME of power transformers is intensely dependent on the ability of the insulation system, composed by solid and liquid dielectrics, to withstand electrical stress. During service, the insulation system is subjected to a combination of stresses such as electrical, chemical, mechanical and thermal ones, which in combination with moisture can leads to weaknesses in the insulation system in which partial discharge (PD) activity can appear [1], [2]. PD monitoring in power transformers is key for evaluating the degradation of its insulation system, more concretely when the transformer is in service. Besides, on-site PD measurements before its commissioning are also of interest to evaluate the insulation system of power transformers in case of being damaged during transportation and deployment in the field [3], [4].

The failure modes for substation transformers with rated voltages higher than $100 \mathrm{kV}$ are mainly due to dielectric, mechanical and electrical issues, see Fig. 1. In addition, more than $40 \%$ of major failures are produced in insulation weaknesses and windings as it is shown in Fig. 2.

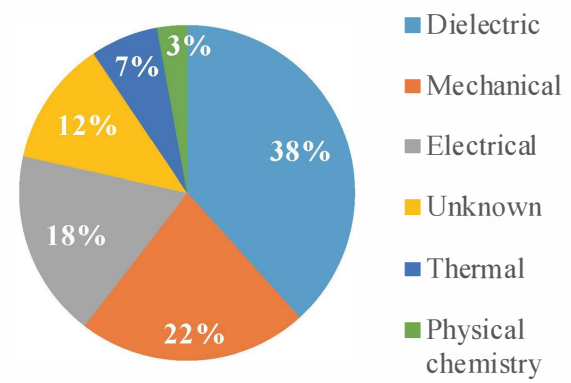

Fig. 1. Failure modes in substation transformers [4]

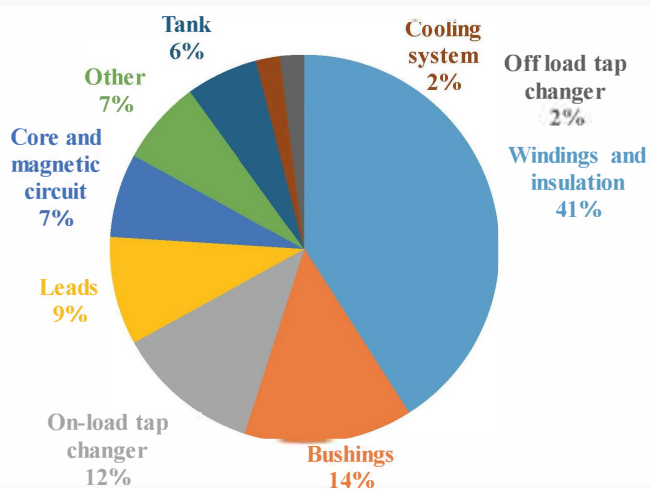

Fig. 2. Failure statistics for power transformers [5].

Due to the key role of power transformers in power grids, it is crucial to assess possible damages in its insulation system. One of the most common ways of prevent outages is testing and evaluating the oil of the transformer. Additionally, during the last decades, the tendency is to investigate which is the remaining life of power transformers also evaluating the PD activity inside this type of electrical asset. According with the information depicted in Fig. 3, around 62000 documents have been published during last years with the keywords "power transformers and partial discharges", specifically in the period 2000-2018. Being $37.5 \%$ of this total taking into account the data up to year 2010. Since 2011, this topic has shown an important growth of interest from the scientific community and industry, counting on a $62.5 \%$ of the total amount of the publications.

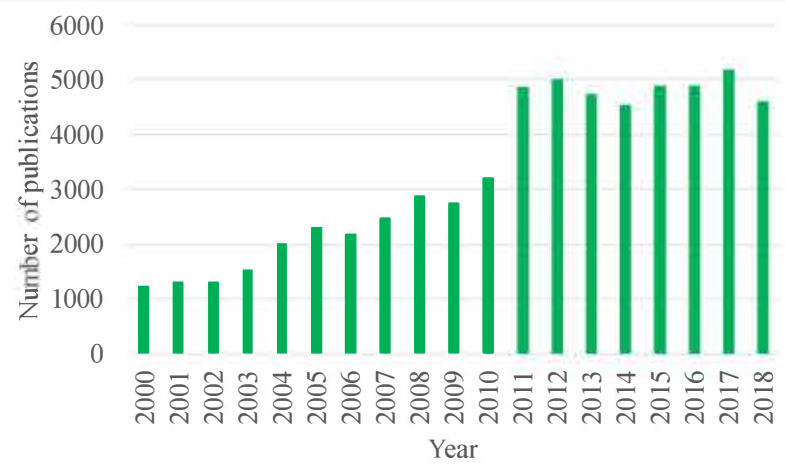

Fig. 3. Published papers in the topic "power transformers and partial discharges" from 2000 to 2018 based on Google Scholar information. 
The different techniques, circuit and sensors for condition monitoring of power transformers can be classified as follows:

- Electrical measurements [3], [5]:

o Indirect circuit using an external coupling capacitor.

- Coupling PD from bushing taps.

- Use of inductive sensors, such as High-Frequency Current Transformers (HFCT).

- Unconventional methods [6] :
- Acoustics.
- Ultra-high-frequency (UHF) acquisitions.

The use of conventional techniques allows identifying if a power transformer has PD activity inside it. With the new unconventional methods, it would be also possible to localize the defect inside the power transformer tank and the sensitivity in the detection can be improved [7]-[9]. With this aim, it is necessary to install sensors on the walls of the tank [10]-[15] and/or in the expansion valve for the oil in the power transformer [16]. The correlation among these techniques [17] is allowing preventing outages of these electrical assets.

On the other hand, during the last two decades, a new focus on condition monitoring based on PD modelling techniques have been studied as a compliment of PD diagnosis [18]-[20] using electrical measurements and equivalent electrical models for windings and PDs.

In this document, the different conventional and unconventional techniques for PD measurements in power transformers are discussed. Besides, a review on PD modelling as an additional tool for condition monitoring is presented.

The paper is organized as follows: in section II, the different methods for measuring PD in power transformers are discussed; in section III, a review about PD models in solid dielectrics as a compliment for PD diagnosis is presented. Finally, some conclusions are drawn in section IV.

\section{METHODS FOR MEASURING PD IN POWER TRANSFORMERS}

When there is PD activity in power transformers, different physical phenomena can be propagated within the transformer, such as conducted currents, ultrasonic propagation in the oil, electromagnetic (EM) waves, even light emission and production of gases such as $\mathrm{H}_{2}$. All these physical effects can be measured to detect PD activity in the transformer.

\section{A. Conventional PD measurements}

Conventional PD measurements in power transformers can be carried out in the factory or in the field and should be performed using an indirect measurement circuit in which a coupling capacitor $C_{k}$ is set in parallel to the insulation system $C_{A}$ under test [3], [18]. The coupling capacitor $C_{k}$ can be replaced by the capacitive bushing according to the testing setup, see Fig. 4. The indirect circuit is more sensitive and easier to implement compared with other acquisition techniques [2]. The apparent charge is measured using a PD instrument connected to a measuring impedance
$Z_{M}$ through the PD pulses will cross. There are standards that establish apparent charge limits for evaluating the equipment condition under tests made in the factory. However, resonances can provoke important errors estimating this parameter, so other parameters such as the number of PDs and its trending during the time must be taken also into account.

For on-site measurements, it is not available to implement the circuit shown in Fig. 4 due to the size and weight of HV coupling capacitors. In case of having a bushing tap in the bushings of the power transformer, acquisitions can be done through them according to Fig. 5.

In any case, the measuring system should be calibrated before any $\mathrm{PD}$ measurement/test is performed. The reading of the PD instrument must be set to the calibration signal. After the installation of a new power transformer, an off-line PD measurement is recommended. The PD test is considered successful if no continuous PD activity is greater than the specified apparent charge, and if there is not any rising trend on it during the duration of the test. It is known that the real charge at the defect site cannot be measured, especially in power transformers. The propagation path influences the PD signals originated within the transformer. The same defect occurring at different places inside the transformer will give different readings in the measuring device. In consequence, the value of the real involved charge cannot be known. Furthermore, in on-line PD measurements the background noise level is usually higher than the admissible level and consequently PD measurements according to the conventional approach [3] (IEC-60270 standard) are not recommended/feasible.

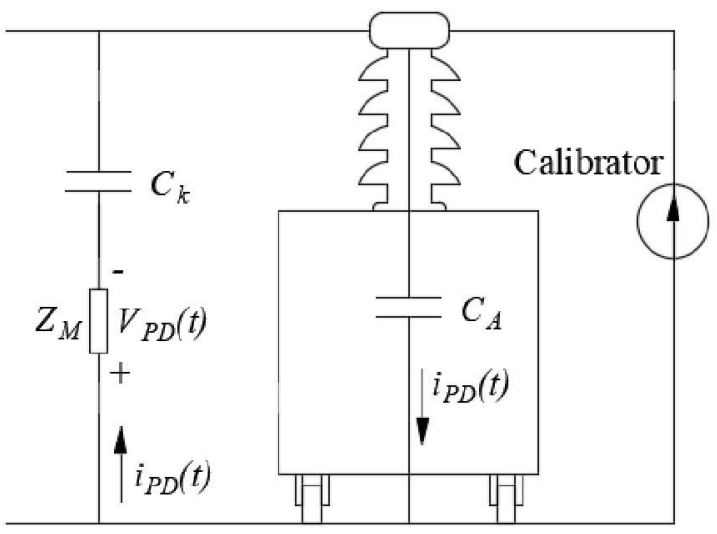

Fig. 4. Indirect measurement circuit according with IEC-60270 standard using an external coupling capacitor, $C_{k}$.

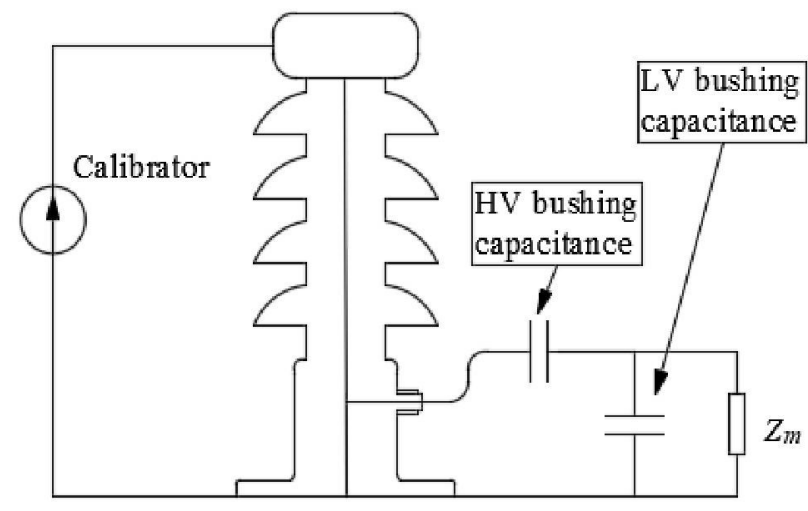

Transformer tank (grounded)

Fig. 5. Coupling PD from bushing taps. 


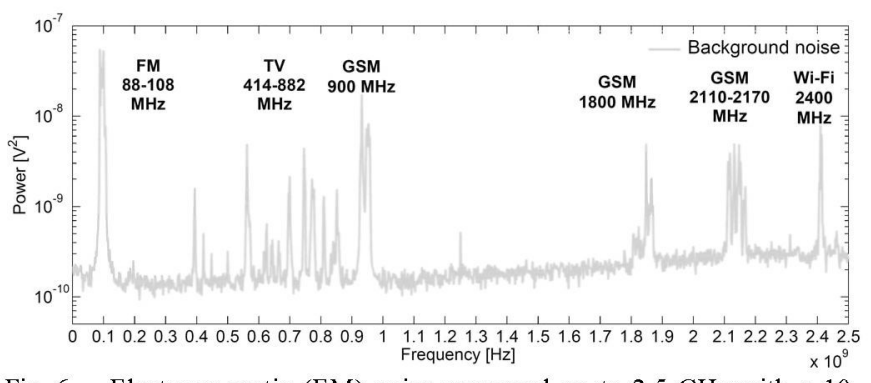

Fig. 6. Electromagnetic (EM) noise measured up to $2.5 \mathrm{GHz}$ with a 10 $\mathrm{cm}$ dipole antenna.

\section{B. Unconventional PD measurements}

Alternatively, non-conventional PD measuring methods can be applied in compliance with IEC-TS-62478 [6] to verify $\mathrm{PD}$ activity inside power transformers during on-line PD measurements. Although, in most cases, PD activity in power transformers can be detected by dissolved gases analysis (DGA), PD monitoring applying EM methods gives complementary information about the various PD sources that can be involved inside or outside the insulation media of the transformer and about the PD trend in real time. These types of defects can occur due to bubbles in oil, cavities in the paper, surface or corona defects and metallic particles floating in the oil [22]. Correlation with parameters such as load power, temperature, and voltage level are very important to identify and locate the defect in the insulation system

Invasive UHF sensors inside the grounded transformer tank are used to achieve a significant level of EM immunity against external disturbances such as FM radio, TV, GSM and Wi-Fi sources among others [23], see Fig. 6.

UHF signals propagating inside the tank present only a moderate attenuation. When applying this last approach, two typical types of UHF sensors can be used: a) an antenna (UHF sensor) to be placed in standardized oil valves DN50/DN80 [16] and b) an UHF plate sensor to be installed in new transformers onto the tank wall [10]-[15]. The last configuration allows localizing the PD source inside the tank by calculating the arrival time from the source to the sensors.

The depth achieved when inserting the antenna UHF sensor inside the oil valve, has a decisive influence on the sensitivity acquired in the measurements. Antenna insertion is often limited to ensure no damage to the active part of the transformer. However, this UHF method is not applicable for power transformers with oil valves different to the standardized ones (DN50/DN80) nor for power transformers in which invasive UHF sensors are not permitted.

The conducted PD signals can be captured using the bushing taps (see Fig. 5), if they are available, or using noninvasive sensors, that operate in the frequency range from hundreds of $\mathrm{kHz}$ to a few megahertz. Radiated UHF signals can be also used to synchronize/trigger PD pulses acquired by the external high frequency (HF) sensors.

Furthermore, on-line PD measurements applying EM methods should take into account the results presented in [24] where the expected spectral content of conducted PD signals corresponding to insulation defects placed at different sites of the winding is lower than few megahertz. Therefore, a measuring frequency range from $0.1 \mathrm{MHz}$ to 10 $\mathrm{MHz}$ can be adequate for external PD sensors, designed to capture conducted PD signals coming from inside the transformer. Although dielectric defect located inside the transformer generates electrical pulses with very fast risetime, which radiate EM waves up to the UHF range (300$3000 \mathrm{MHz}$ ), the spectral content of conducted PD signals after traveling through the transformer windings reduces drastically.

As it was mentioned before, the use of invasive UHF sensors is not always possible, one chance is to install a UHF sensor on the base of the transformer bushing [25], where EM radiation can go out, together with an inductive sensor, HFCT or measuring impedance, performing the acquisitions using the test taps of bushings. In case the power transformer is cable-connected with insulated power cables, it is possible to use additional HFCTs on the ground connection of each cable sheath. These additional sensors allow discriminating from where, inside or outside the transformer, it is coming the PD activity [26]. The synchronized PD signals are measured with all distributed sensors (antennas and HFCT) and automatically detected without any threshold level adjustment, because the background noise is suppressed using an advanced filtering tool based on the wavelet transform with a statistical signal treatment [25]. Finally, it is possible to localize the PD source position of the insulation defects determining the time delay between two PD pulses generated by the same PD source traveling towards two sensors placed in different positions [25], [27]. A GPS signal is used for synchronizing all the acquisitions.

Other type of unconventional technique is the one that acquire the acoustic emission (AE), also reported by other authors as ultrasound/ultrasonic propagation, of $\mathrm{PD}$ through oil via acoustic sensors, which have a narrowband detection at $150 \mathrm{kHz}$ [28]. Besides, by using, at least four sensors, it would be possible to localize PD sources in the transformers, e.g. using triangulation algorithms among others [29].

These sensors can be installed externally on the tank wall. However, their sensitivities are very low due to the attenuation of the acoustic waves propagation through the oil and have the same problem that the UHF method with regard to multi-path propagation. A better performance of the sensor can be obtained if it is installed inside the tank, but further studies must be done to explore the accelerated aging of the sensor immersed in the transformer environment for long time exposures. In this sense, new optical sensors [30] can be considered to be installed inside the transformer tank for measuring the light propagated by PD activity. Some advantages of these sensors are its broad response spectral range (from ultraviolet to near infrared), high EMinterference immunity, and a high level of integration. Currently, this type of technology has been proven as builtin optical PD monitoring in gas insulation systems [31] and it would require a step forward for being applied in power transformers in near future.

\section{PD MODELLING AS A COMPLEMENTARY TOOL FOR PD DIAGNOSIS}

As it was previously discussed, there are different methods for measuring PD sources in power transformers. However, it is difficult to determine the characteristics of the cavities where PDs activity is taking place [32]. Changes in PD patterns due to aging can be predicted through simulation of cavities with changes in pressure, gas content, 
TABLE I

Winding Models For PD PROPAGATION AND PD LOCATION ANALYSIS

\begin{tabular}{|c|c|c|}
\hline Model & Characteristics & References \\
\hline Capacitive & $\begin{array}{l}\text { Simple implementation. Valid for a } \\
\text { limited frequency range, few hundred of } \\
\mathrm{kHz} \text {. For accuracy improvement, the } \\
\text { capacitances in the model should be } \\
\text { calculated from construction data, } \\
\text { which is sometimes impossible. This } \\
\text { method is inadequate for modelling } \\
\text { continuous disc winding. }\end{array}$ & [43], [44] \\
\hline $\begin{array}{l}\text { Detailed } \\
\text { ladder } \\
\text { network }\end{array}$ & $\begin{array}{l}\text { This is a distributed parameters model } \\
\text { with resistance, inductance, capacitance } \\
\text { and conductance per unit of length for } \\
\text { each section of the winding. It is valid } \\
\text { for a frequency range up to tens of } \\
\text { MHz. However, it not considers the PD } \\
\text { detection impedance and it is } \\
\text { inadequate for studying the PD } \\
\text { propagation. }\end{array}$ & [45] \\
\hline $\begin{array}{l}\text { Multi- } \\
\text { conductor } \\
\text { transmission } \\
\text { line }\end{array}$ & $\begin{array}{l}\text { Uses the distributed parameters } \\
\text { transmission line model, similar to } \\
\text { ladder network. However, in order to } \\
\text { improve the accuracy and applicability } \\
\text { to the MHz frequency range, all mutual } \\
\text { couplings, inductive and capacitive, } \\
\text { with every turn are included. This } \\
\text { model requires higher computational } \\
\text { efforts and the time solution is longer. }\end{array}$ & {$[46],[47]$} \\
\hline Hybrid & $\begin{array}{l}\text { It combines the detailed ladder network } \\
\text { and multi-conductor transmission line } \\
\text { models. It is valid in the range of tens } \\
\text { of kHz to hundreds of } \mathrm{MHz}(10 \mathrm{kHz} \text { to } \\
\text { a few } \mathrm{MHz}) \text {. They use a Black Box } \\
\text { model approach in which models are } \\
\text { synthesized from direct measurements } \\
\text { taken on the complete transformer, or } \\
\text { reduced by using time-domain and } \\
\text { frequency-domain sensitivity } \\
\text { techniques. They are not available in } \\
\text { the design stage. Computational } \\
\text { capabilities are reduced. }\end{array}$ & [48], [49] \\
\hline
\end{tabular}

surface conductivity and temperature, among others [33]. When the measured signals are available, they can be evolved to determine the optimal values of circuit parameters in the model [34] and at the same time, as a diagnosis tool, this allows to determine the characteristics of the insulation, such as permittivity, conductivity and void geometry. In addition, with real PD data, it is possible to use pattern validation techniques for determining defect characteristics [35].

On the other hand, the analysis of the simulated signals allows determining their characteristic parameters in magnitude, waveform and frequency content and relating them to required characteristics of the acquisition system [36].

Simulate the PD propagation in power transformers is a hard task because power transformers have complicated multi-material insulation systems [37]. Some studies in distribution transformers insulated with oil and dry-type use the PD pulse and the analytical solutions to equivalent circuits of winding models to study the propagation characteristics of a PD [38]. However, their conclusions are not general and need additional experimental validation.

When electrical methods are used for locating PD sources, it is necessary to model the PD in the insulation system and the transformer windings through equivalent electric circuits.

Three models have been widely used for simulating PDs in solid dielectrics [39]; namely, Finite-Element Analysis
(FEA) models, analytical models and three-capacitance or " $a b c$ " models. The three-capacitance is the most used model for PD simulation at different conditions [40]. In [41] a method based on wavelet transformation for PD location on transformer windings was presented. The detailed transformer-winding model and " $a b c$ " model were simulated using Electromagnetic Transients Program (EMTP) tools for determining the current in neutral point when defects are located at different points in the winding. The method allows locating PD emitters in the coil-to-coil insulation and was validated and showed acceptable agreement when compared with experimental data.

On the other hand, Multi-conductor Transmission Line (MTL) models, capacitive networks models, detailed parameter models and hybrid models have been used for transformer winding modelling depending on the operating frequency and the winding type [37]. However, due to the stochastic behaviour of the PD phenomena and the complex structure of the insulation system is difficult to reproduce an accurate model of the winding [42]. Table I, summarizes some characteristics of the winding models for PD propagation modelling and PD location.

\section{CONCLUSIONS}

This paper discusses different PD measuring approaches developed for power transformers. Conventional and unconventional methods for PD measuring were analysed, showing their advantages, disadvantages and future development trends. Unconventional methods, such as, synchronized PD measurements performed by means of noninvasive HFCT sensors and UHF sensors combined with a powerful filtering tool allows the identification and localization of the PD sources without using invasive sensors.

The analysis of PD amplitude, polarity and time delay of the pulses detected with various PD sensors used during online PD tests, permits to identify whether the PD source is inside or outside the transformer.

In the case of antennas installed in the walls of the power transformer tank, it would be possible to localize PD sources inside the power transformer calculating the arrival time from the source to the sensors.

Additionally to this, PD modelling can be used as a compliment to electrical measurements of PD for locating PD sources in the windings and evaluate the operating conditions along the lifetime of the power equipment.

\section{ACKNOWLEDGMENT}

Authors would like to thank X. M. Lopez-Fernandez for proposing the interesting issue of reviewing about the importance of PD monitoring of power transformers. 